\title{
Sestrin 2 and sestrin 3 suppress NK-92 cell-mediated cytotoxic activity on ovarian cancer cells through AMPK and mTORC1 signaling
}

\author{
Xuejin Wang ${ }^{1, *}$, Weifeng Liu ${ }^{2,}{ }^{,}$, Deyi Zhuang ${ }^{3}$, Shaoxian Hong ${ }^{4}$ and Jingfang Chen ${ }^{5}$ \\ ${ }^{1}$ Department of Reproductive Medicine, The Second Affiliated Hospital of Fujian Medical University, Quanzhou 362000, China \\ ${ }^{2}$ Department of Anesthesiology, The Second Affiliated Hospital of Fujian Medical University, Quanzhou 362000, China \\ ${ }^{3}$ Department of Neonatal, Children's Hospital of Fudan University Xiamen Branch (Xiamen Children's Hospital), Xiamen \\ 361000, China \\ ${ }^{4}$ Department of Pediatric Intensive Care Unit, Children's Hospital of Fudan University Xiamen Branch (Xiamen Children's \\ Hospital), Xiamen 361000, China \\ ${ }^{5}$ Department of Pediatrics, Children's Hospital of Fudan University Xiamen Branch (Xiamen Children's Hospital), Xiamen \\ 361000, China \\ *These authors have contributed equally to this work
}

Correspondence to: Jingfang Chen, email: jfchen01@126.com

Keywords: NK cells, ovarian cancer, sestrin, MTOR, AMPK

Received: July 18, $2017 \quad$ Accepted: August 29, $2017 \quad$ Published: October 04, 2017

Copyright: Wang et al. This is an open-access article distributed under the terms of the Creative Commons Attribution License 3.0 (CC BY 3.0), which permits unrestricted use, distribution, and reproduction in any medium, provided the original author and source are credited.

\section{ABSTRACT}

Ovarian cancer is one of the major cancer types. NK-92 cell line, which has consistently and reproducibly high anti-tumor cytotoxicity, may be used for immunotherapy against ovarian cancer. Understanding the mechanisms that regulate the anti-tumor activity of NK-92 cells is important for developing novel therapeutic strategies. In the current study, using an ovarian cancer xenograft mouse model, we identified the up-regulation of sestrin 2 (SESN2) and sestrin3 (SESN3) in intratumoral NK-92 cells. Lentivirus-transduced NK-92 cells, which overexpressed SESN2 or SESN3 after doxycycline treatment, exhibited less expression of activating receptors, perforin and granzyme B. Overexpression of SESN2 and SESN3 impaired tumoricidal effect of NK-92 cells both in vitro and in vivo. Furthermore, overexpression of SESN2 and SESN3 inhibited mTORC1 signaling while promoting AMPK signaling in NK-92 cells. Taken together, our data highlights the crucial effects of SESN2 and SESN3 on NK-92 cell-mediated anti-ovarian cancer activity. This study might be valuable for designing a novel therapeutic strategy for ovarian cancer.

\section{INTRODUCTION}

Ovarian cancer is the one of the most common cancer in women all over the world. Although surgery and chemotherapy are available, the 5-year survival rate is still relatively low. Immunotherapy could be a promising therapeutic approach since ovarian cancer is an immunogenic tumor that can be recognized by the host immune system [1]. Recently NK-92 cell line has shown promising tumoricidal potency and therefore has undergone intensive study [2]. NK-92 cell line is easy to be genetically modified to distinguish certain tumor antigens or to promote the activity of monoclonal antibodies via antibody-dependent cytotoxicity [3]. Currently it is the only NK cell line which has undergoing clinical trials [4-6]. In addition, NK-92 cell line has been shown to kill ovarian cancer cells including OVCAR-3 [7-9]. However, the molecular mechanisms by which NK-92 cell activity 
is regulated in ovarian cancer remains unclear. NK cells might reduce or even lose their cytotoxic activity against tumor cells in some tumor patients $[10,11]$, due to altered expression of activating receptors and inhibitory receptors.

Sestrins (SESNs) are a family of highly conserved proteins that are induced upon various conditions of stress, including DNA damage and oxidative stress [12]. Their functions have not been well documented. SESN1 and SESN2 are intracellular leucine sensors and p53 target genes that restrain mTORC1 signaling $[13,14]$. SESN3 enhances hepatic insulin sensitivity through activation of mTORC2-Akt signaling [15], and it also inhibits mTORC1 [16]. Due to the importance of mTORC1 for immune cell fucntions $[17,18]$, it is likely that SESNs impact immune reactions. Indeed, SESNs inhibit T cell immunity [19], maintain macrophage survival and inhibit inflammatory response [20, 21]. However, the significance of SESNs for NK cell development and function has not been addressed.

In this study, using an ovarian cancer xenograft mouse model and NK-92 cell line, we identified the upregulation of SESN2 and SESN3 in intratumoral NK-92 cells. To determine the roles of SESN2 and SESN3 in the regulation of NK-92 cell activity, we established a lentivirus-mediated SESN inducible expression system in NK-92 cells. Lentivirus-transduced NK-92 cells, which expressed SESN2 or SESN3 upon doxycycline treatment, down-regulated expression of NKG2D and NKp44, perforin and granzyme B, and their tumoricidal activity was subsequently impaired both in vitro and in vivo. Furthermore, overexpression of SESN2 and SESN3 inhibited mTORC1 signaling while promoting AMPK signaling in NK-92 cells. Taken together, our data highlights the crucial effects of SESN2 and SESN3 on NK-92 cell-mediated anti-ovarian cancer activity.

\section{RESULTS}

\section{Intratumoral NK-92 cells up-regulate SESN2 and SESN3 expression}

We firstly tested NK-92 cell activity in a subcutaneous OVCAR-3 xenograft model. CD $45^{+} \mathrm{CD} 56^{+}$ NK-92 cells were sorted from the spleen, peritoneum and tumor grafts of the tumor-bearing mice using flow cytometry. Notably, intratumoral NK-92 cells were divided into 2 subgroups according to their CD56 expression profile: CD56 ${ }^{\mathrm{hi}}$ and CD56 ${ }^{\mathrm{dim}}$, while splenic and peritoneal NK-92 cells were uniformly CD56 ${ }^{\text {hi }}$ (Figure 1A). Although we did not observe a significant change in tumor volume (data not shown), we unintentionally found higher expression of SESN2 and SESN3 in intratumoral NK-92 cells than those in splenic and peritoneal NK-92 cells (Supplementary Figure 1 and Figure 1B). CD56 ${ }^{\text {hi }}$

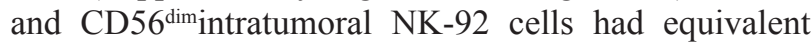
expression of SESN2 and SESN3, whereas SESN1 expression was not dramatically changed (Figure 1B
\& 1C). In addition, expression of SESN2 and SESN3 in normal human blood NK cells were extremely low (Supplementary Figure 1). The up-regulation of SESN2 and SESN3 in intratumoral NK-92 cells was also observed in another subcutaneous ovarian cancer model using SKOV3 cells (Supplementary Figure 2).

\section{SESN2 and SESN3 suppresses NK-92 cell activation}

To explore the effects of SESN2 and SESN3, we established NK-92 cell lines expressing inducible SESN2 and SESN3, respectively (Supplementary Figure $3)$. Treatment with doxycycline remarkably induced expression of GFP and SESNs in these cells (Figure 2A \& 2B). In addition, expression of two NK cell activating receptors, NKp44 and NKG2D, was significantly decreased on SESN2 or SESN3-overexpressing NK92 cells (Figure 2C \& 2D). Expression of other two NK activating receptors, Nkp30 and NKp46, were not profoundly changed (Supplementary Figure 4). The cell viability was not impacted by overexpression of SESN2 or SESN3 (Supplementary Figure 5).

\section{SESN2 and SESN3 suppresses cytotoxic activity of NK-92 cells in vitro}

Lentivirus-transduced NK-92 cells were co-cultured with OVCAR-3 cells to evaluate cytotoxicity. SESN2 or SESN3-overexpressing NK-92 cells induced less lysis and apoptosis of OVCAR-3 cells, as compared with NK-92 cells not expressing SESNs (Figure 3A \& 3B). Furthermore, expression of perforin and granzyme B, which are cytotoxic mediators, were profoundly reduced in SESN2 or SESN3-overexpressing NK-92 cells, in comparison with other groups (Figure 3C \& 3D). Another cytotoxic factor, TNF- $\alpha$, was moderately decreased in SESN2 or SESN3-overexpressing NK-92 cells (Figure $3 \mathrm{E})$.

\section{SESN2 and SESN3 inhibits mTORC1 signaling and promotes AMPK signaling}

SESNs can suppress mTORC1 signaling [16, 22, 23]. To evaluate if this happened in NK-92 cells, activating phosphorylation of mTOR and 4EBP1 was determined. As shown in Figure 4A \& 4C, after doxycycline treatment, phosphorylation of mTOR and 4EBP1 was remarkably decreased in SESN2 or SESN3-overexpressing NK-92 cells, while the protein levels of mTOR and 4EBP1 were not changed. Since AMPK signaling suppresses mTORC1 signaling [24], and SESNs can enhance AMPK signaling $[25,26]$, we checked AMPK activation and found that SESN2 or SESN3-overexpressing NK-92 cells expressed more phosphorylated AMPK $\alpha$ and AMPK $\beta 1$, in comparison with other groups (Figure 4B \& 4D). Interestingly, the total 
AMPK $\beta 1$ protein was increased in SESN2 or SESN3overexpressing NK-92 cells (Figure 4E). To determine the effect of AMPK signaling on cytotoxic activity of NK-92 cells, SESN2 or SESN3-overexpressing NK-92 cells were treated with AMPK inhibitor Compound $\mathrm{C}$ before co-culture with OVCAR-3 cells. Compound C inhibited AMPK $\alpha$ activation and promoted mTORC1 signaling (Supplementary Figure 7), and subsequently restored cytotoxic activity of SESN2 or SESN3-overexpressing NK-92 cells (Figure 4F).

\section{SESN2 and SESN3 suppresses NK-92 cell activity in vivo}

To determine effects of SESN2 and SESN3 in vivo, lentivirus-transduced NK-92 were pre- treated with doxycycline and transferred into mouse peritoneum. Two days later, NK-92 cells were isolated from the peritoneal cavity based on CD56 expression (Figure 5A). The proportions of SESN2 or SESN3overexpressing NK-92 cells in total peritoneal cells were similar to the proportions of NK-92 cells not overexpressing SESNs (Figure 5B). Additionally, SESN2 or SESN3-overexpressing NK-92 cells showed more phosphorylated AMPKaand less phosphorylated mTOR, as compared with other groups (Figure 5C \& 5D). Moreover, granzyme B expression was significantly lower in SESN2 or SESN3-overexpressing NK-92 cells (Figure 5E). Perforin expression was not altered (Figure $5 \mathrm{E})$.
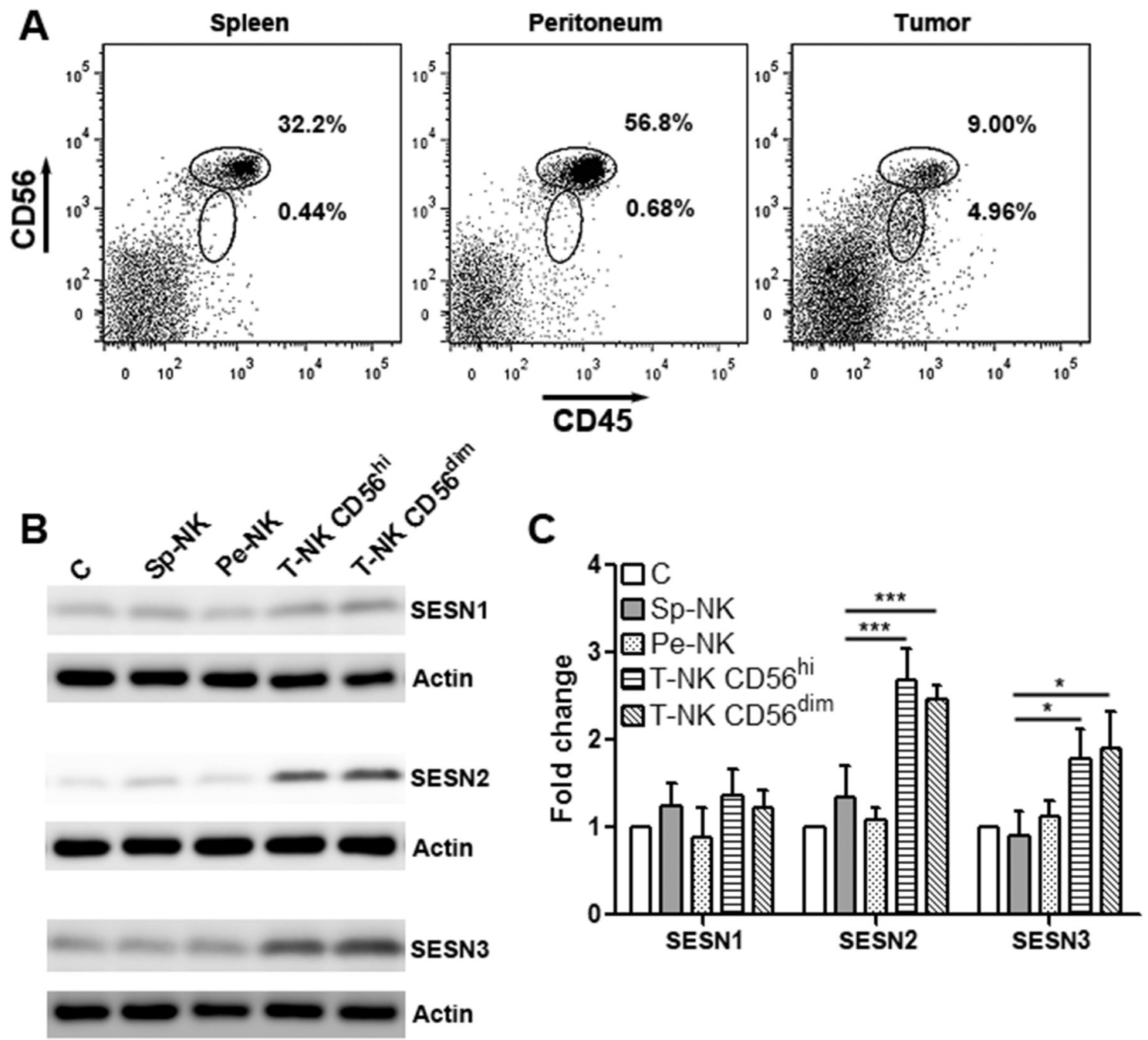

Figure 1: Intratumoral NK-92 cells up-regulate SESN2 and SESN3 expression. (A) Representative flow cytometry plots showing CD45 ${ }^{+} \mathrm{CD} 56^{+}$NK-92 cells in the spleens, peritoneal cavity and tumor xenografts. NK-92 cells were adoptively transferred into tumor-bearing mice through intraperitoneal injection and were then isolated from indicated tissues based on CD45 and CD56 expression. (B) SESN1, SESN2 and SESN3 protein levels in NK-92 populations isolated from different tissues. C: in vitro cultured NK-92 cells. Sp-NK: NK-92 cells isolated from spleens. Pe-NK: NK-92 cells isolated from peritoneal cavity. T-NK C56 int intratumoral NK-92 cells expressing high CD56. T-NK CD56 ${ }^{\mathrm{dim}}$ : intratumoral NK-92 cells expressing dim CD56. (C) Statistics for (B). N= 5 per group. ${ }^{*}, \mathrm{p}<0.05$; ${ }^{* * *}, \mathrm{p}<0.001$. 
A

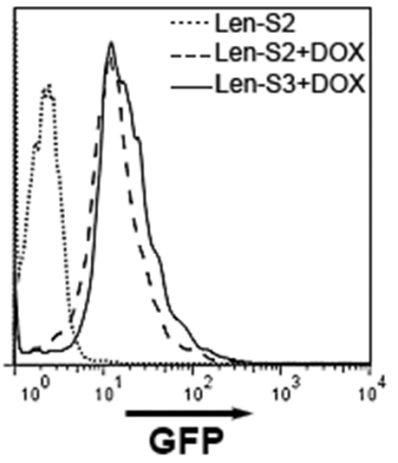

B

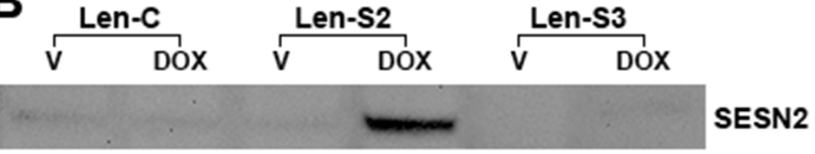

SESN3

Actin

C
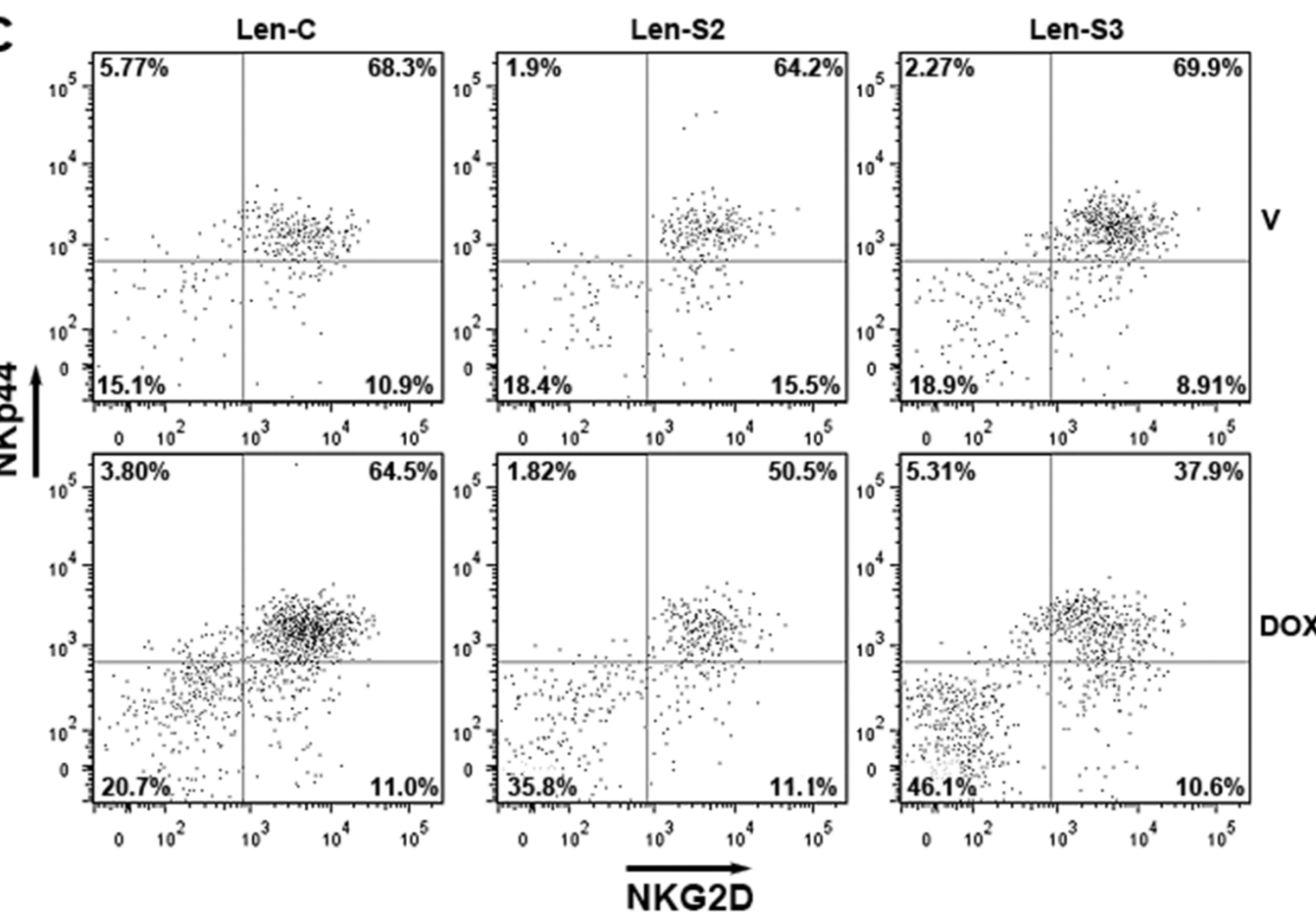

D

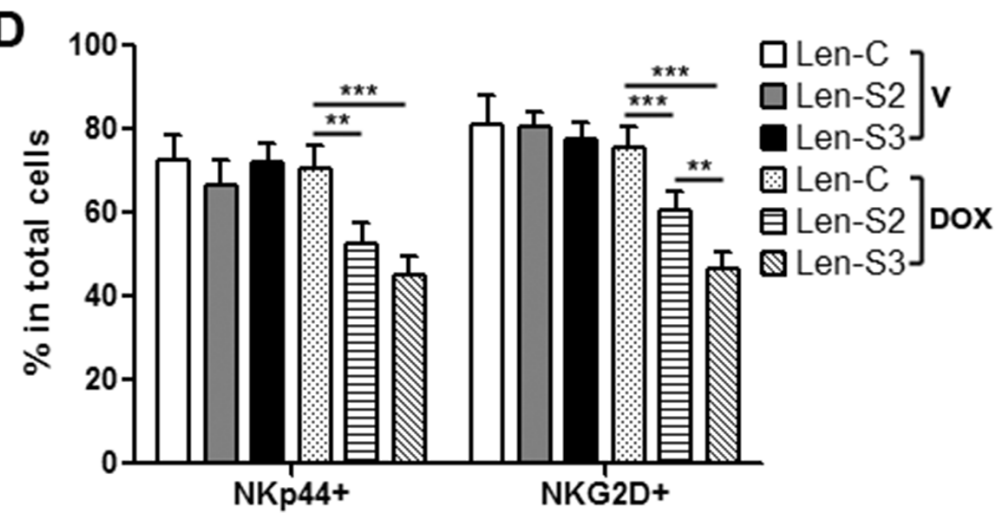

Figure 2: SESN2 and SESN3 expression inhibits NK-92 cell activation in vitro. (A) GFP expression in lentivirus-transduced NK-92 cells with or without doxycycline treatment for $48 \mathrm{~h}$. Len-S2: NK-92 cells transduced with Len-S2. Len-S3: NK-92 cells transduced with Len-S3. DOX: $200 \mathrm{ng} / \mathrm{ml}$ doxycycline. This is a representative image of three independent experiments. (B) SESN2 and SESN3 protein levels in doxycycline-treated NK-92 cells. Len-C: NK-92 cells transduced with lentivirus containing no SESN sequence. Len-S2: NK-92 cells transduced with Len-S2. Len-S3: NK-92 cells transduced with Len-S3. This is a representative image of two independent experiments. (C \& D) Expression of NKp44 and NKG2D on NK-92 cells after treatment with vehicle (PBS) or doxycycline. Representative dot plots were shown in (C). Statistics was shown in (D). V: vehicle. DOX: doxycycline. $\mathrm{N}=6 \sim 8$ per group. ${ }^{*}, \mathrm{p}<0.05 ;{ }^{* *}, \mathrm{p}<0.001 ;{ }^{* * *}$, $\mathrm{p}<0.001$. 
A

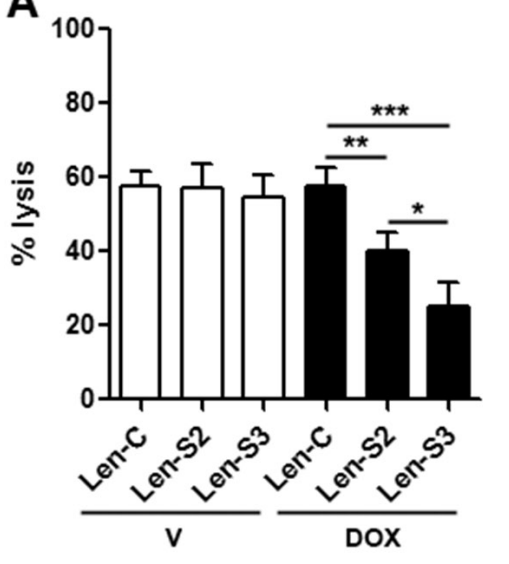

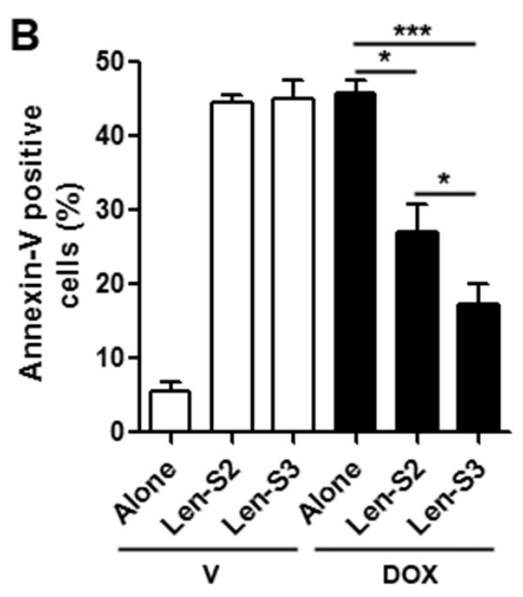
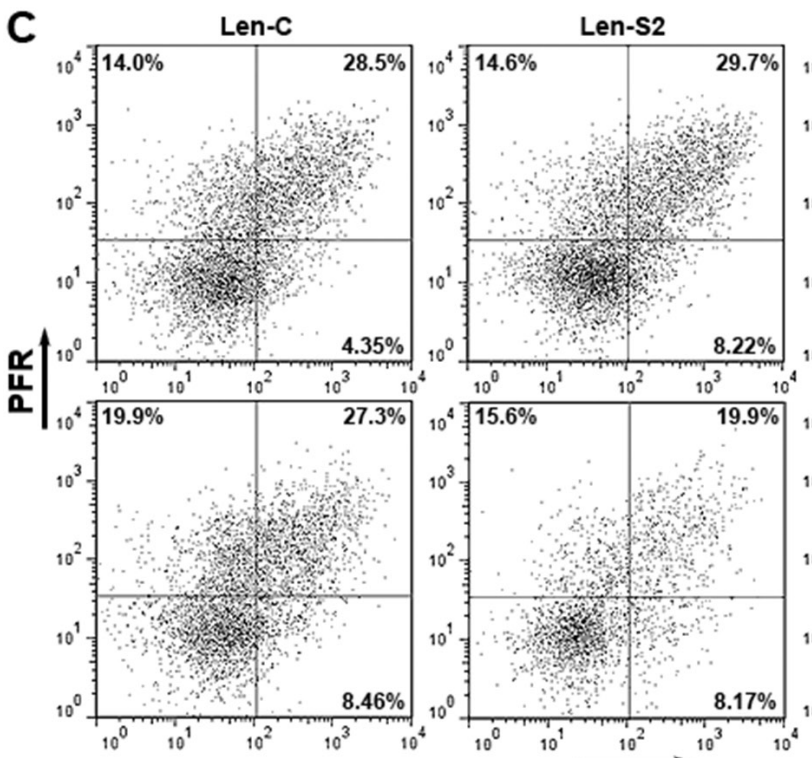

Len-S3
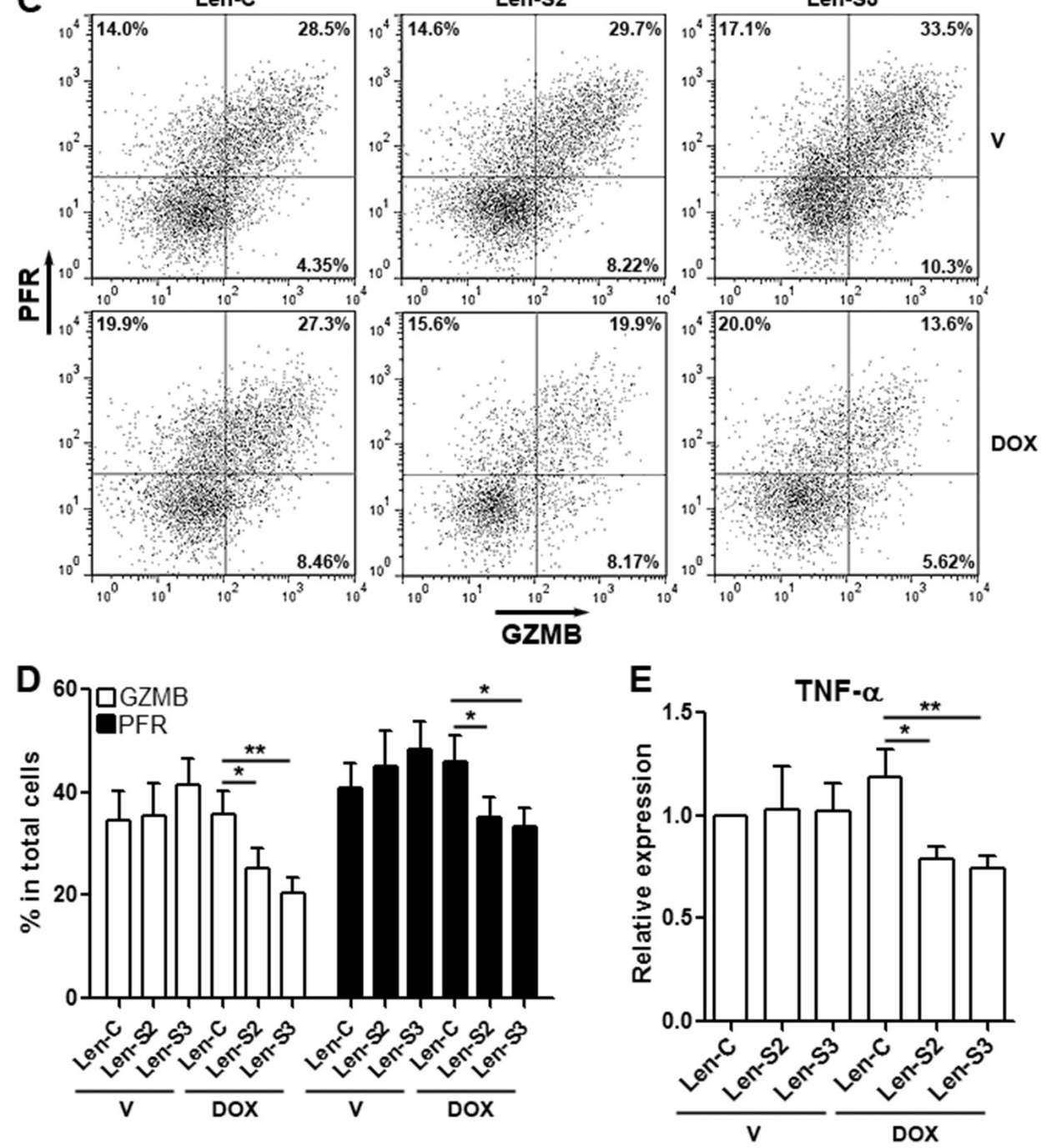

Figure 3: SESN2 and SESN3 expression suppresses NK-92 cell cytotoxic activity in vitro. (A) OVCAR-3 cell lysis after coculture with NK-92 cells. Lenitvirus-transduced NK-92 cells were pre-treated with vehicle or doxycycline for $48 \mathrm{~h}$ prior to co-culture with OVCAR-3 cells. V: vehicle. DOX: doxycycline. Len-C: NK-92 cells transduced with lentivirus containing no SESN sequence. Len-S2: NK-92 cells transduced with Len-S2. Len-S3: NK-92 cells transduced with Len-S3. N=6 per group. (B) OVCAR-3 cell apoptosis after co-culture with NK-92 cells. OVCAR-3 cells were distinguished from NK-92 cells via CD56 staining (Supplementary Figure 6). N=8 per group. (C \& D) Expression of perforin (PFR) and granzyme B (GZMB) in lentivirus-transduced NK-92 cells after vehicle or doxycycline treatment. Representative dot plots were shown in (C), and statistics was shown in (D). N=5 per group. (E) Relative mRNA abundance of TNF- $\alpha$ in lentivirus-transduced NK-92 cells. $N=3$ per group. ${ }^{*}, \mathrm{p}<0.05{ }^{* * *}, \mathrm{p}<0.001{ }^{* * *}, \mathrm{p}<0.001$. 


\section{SESN2 and SESN3 inhibits tumoricidal effect of NK-92 cells in vivo}

To evaluate the tumoricidal activity of NK-92 cells in vivo, we conducted a peritoneal OVCAR-3 xenograft model based on a previous study [27]. OVCAR-3 cells were inoculated in the peritoneal cavity of NOD/SCID/ $\gamma \mathrm{c}^{-/-}$mice which lack mature NK cells. NK-92 cells, pre- treated with or without doxycycline, were injected into the peritoneal cavity 2 days after inoculation. Four days later, peritoneal NK-92 cells and OVCAR-3 cells were analyzed. As shown in Figure 6A \& 6B, in comparison with mice not receiving NK-92 cells, mice receiving vehicle-treated NK-92 cells had less EpCAM ${ }^{+}$OVCAR-3 cells, suggesting that NK-92 cells killed OVCAR-3 cells in vivo. However, in mice receiving doxycycline-treated
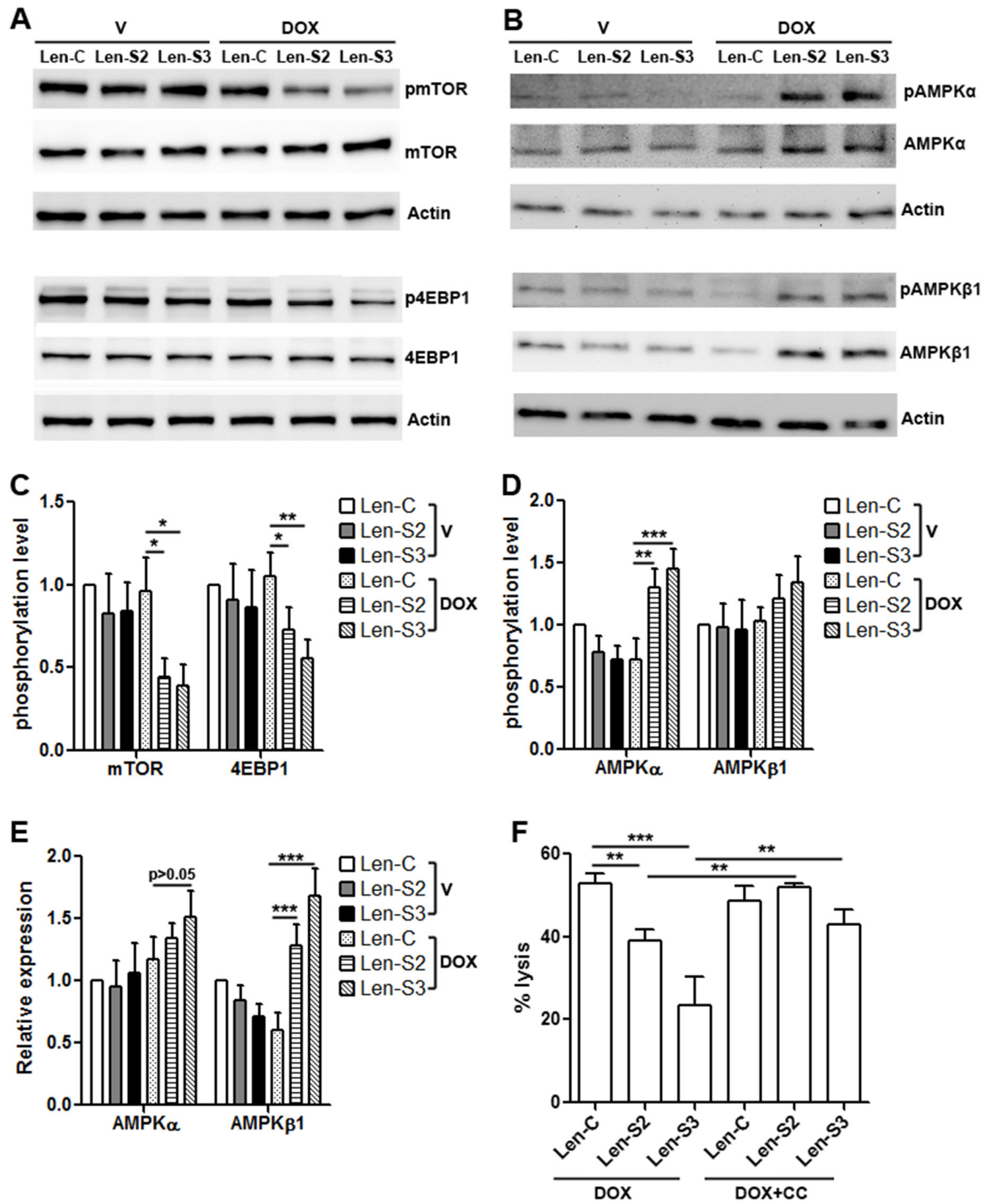

Figure 4: SESN2 and SESN3 expression inhibits mTORC1 signaling and promotes AMPK signaling. (A) Representative Western blot images of phosphorylation of mTOR and 4EBP1 in lentivirus-transduced NK-92 cells. (B) Representative Western blot images of phosphorylation of AMPK $\alpha$ and AMPK $\beta 1$ in lentivirus-transduced NK-92 cells. (C) Statistics for phosphorylation of mTOR and 4EBP1. N=4 per group. (D) Statistics for phosphorylation of AMPK $\alpha$ and AMPK $\beta 1$. N=3 per group. (E) Statistics for total AMPK $\alpha$ and AMPK $\beta 1$ proteins. $\mathrm{N}=3$ per group. (F) OVCAR-3 cell lysis after co-culture with NK-92 cells. Lenitvirus-transduced NK-92 cells were pre-treated with doxycycline and compound $\mathrm{C}$ before co-culture with OVCAR-3 cells. DOX: doxycycline. DOX $+\mathrm{CC}$ : doxycycline with compound C. $\mathrm{N}=8$ per group. ${ }^{*}, \mathrm{p}<0.05 ;{ }^{* *}, \mathrm{p}<0.001 ;{ }^{* * *}, \mathrm{p}<0.001$. 

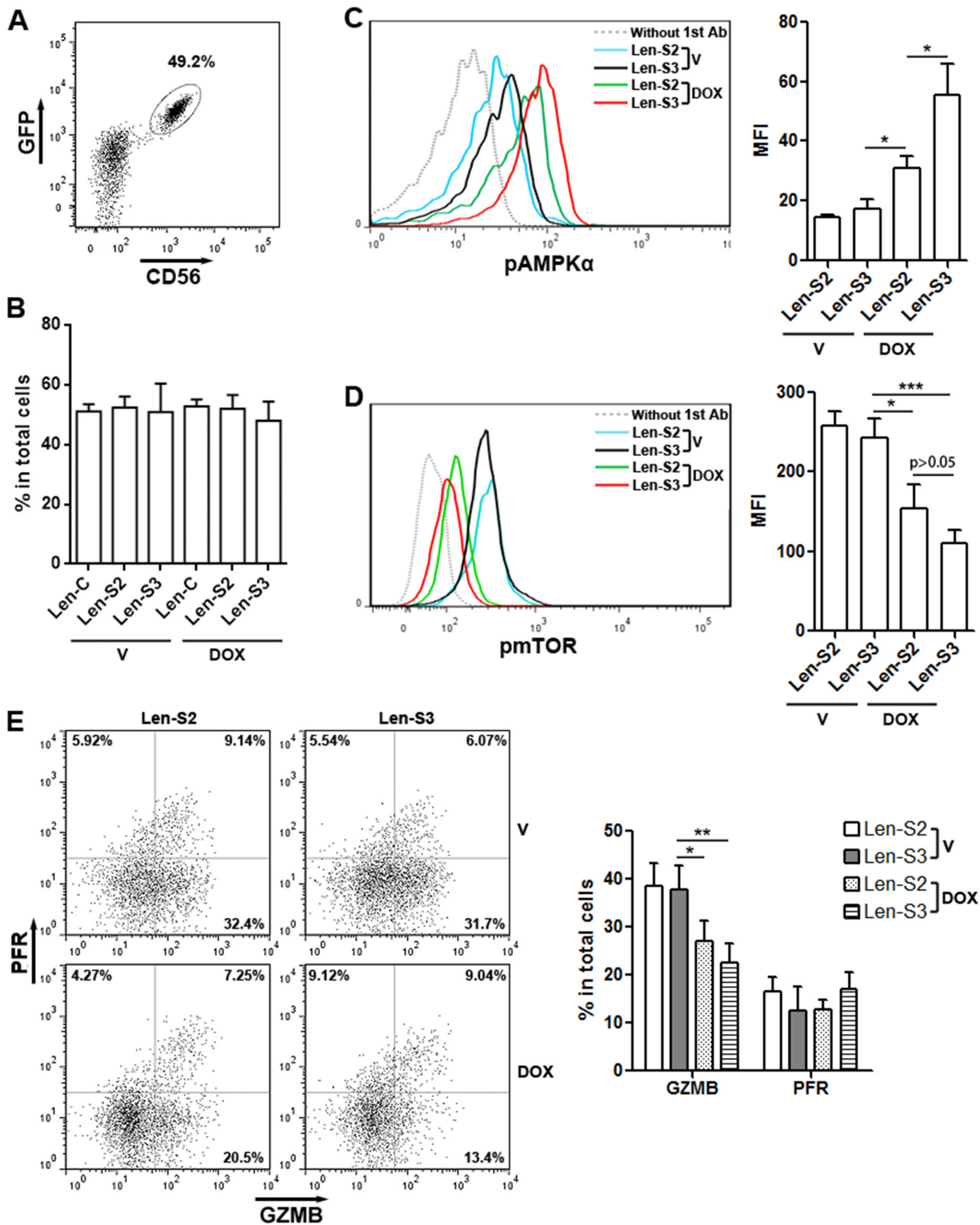

Figure 5: SESN2 and SESN3 inhibit NK-92 cell activity in vivo. (A) Representative dot plot showing the expression of GFP and CD56 in Len-S2-transduced NK-92 cells isolated from peritoneal cavity after doxycycline pre-treatment and adoptive transfer. (B) Proportions of SESN2 or SESN3-overexpressing NK-92 cells and control NK-92 cells in total peritoneal cells on day 2 after adoptive transfer. V: vehicle. DOX: doxycycline. Len-C: NK-92 cells transduced with lentivirus containing no SESN sequence. Len-S2: NK-92 cells transduced with Len-S2. Len-S3: NK-92 cells transduced with Len-S3. N=3 per group. (C \& D) Phosphorylated AMPK $\alpha$ (C) and mTOR (D) in transferred NK-92 cells was detected using flow cytometry. Left panels: representative histograms. Right panels: statistics. N=4 per group. (E) Expression of perforin (PFR) and granzyme B (GZMB) in transferred NK-92 cells. Left panel: representative dot plots. Right panel: statistics. $\mathrm{N}=5$ per group. ${ }^{*}, \mathrm{p}<0.05 ;{ }^{* *}, \mathrm{p}<0.001 ;{ }^{* * *}, \mathrm{p}<0.001$. 
A

No tumor

Tumor

Len-C+V

Len-C+DOX
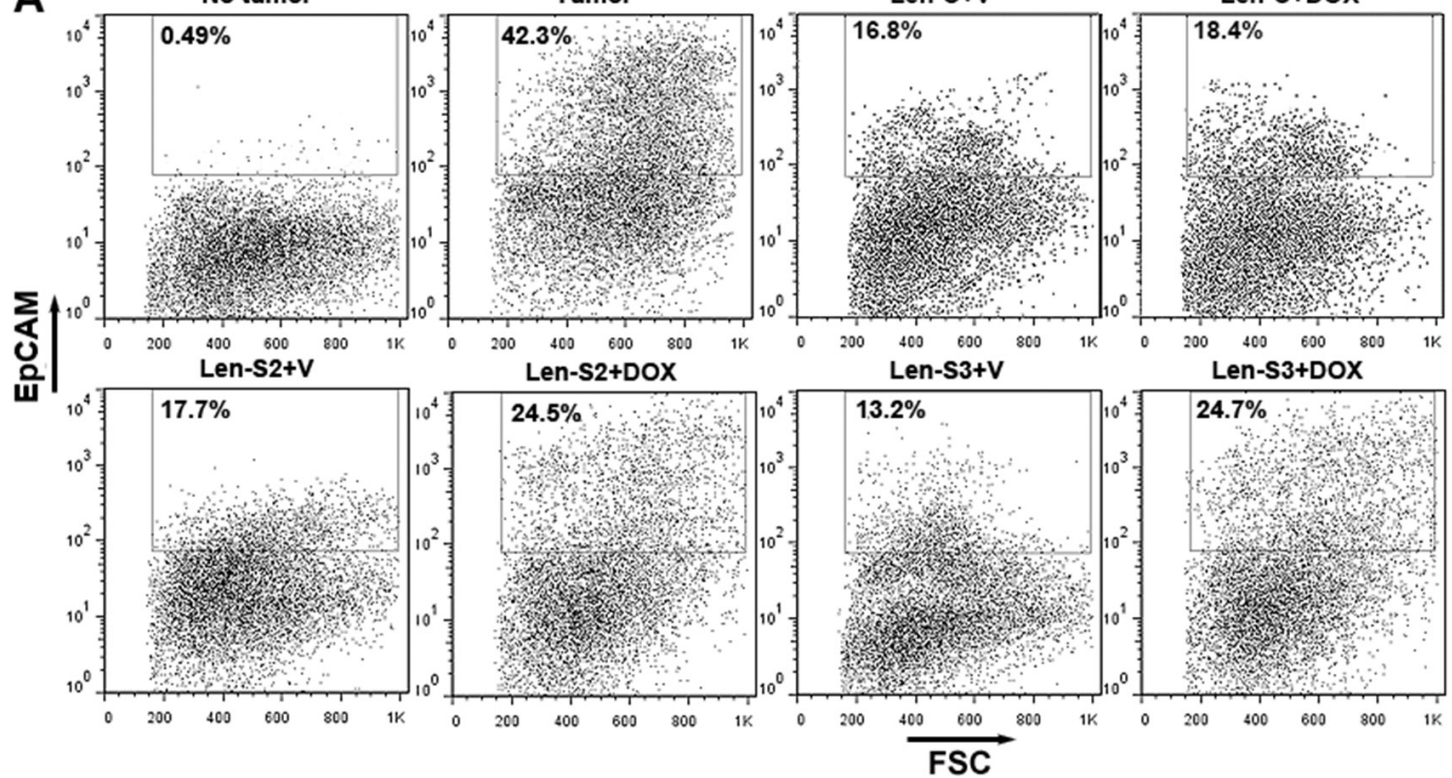

B

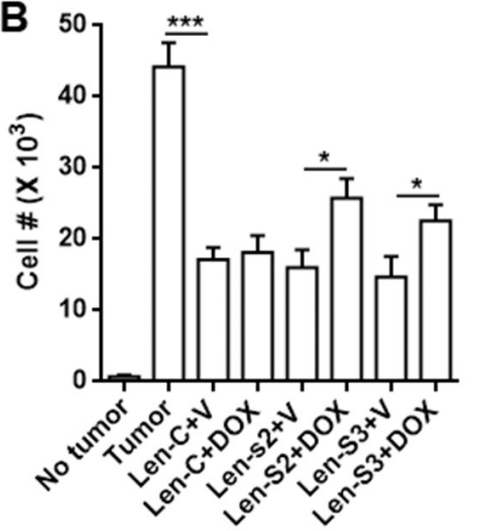

C $\begin{array}{cccccc}\text { Tumor Len-C } & \text { Len- } C & \text { Len-S2 } & \text { Len-S2 } & \text { Len-S3 } & \text { Len-S3 } \\ +\mathrm{V} & +\mathrm{DOX} & +\mathrm{V} & +\mathrm{DOX} & +\mathrm{V} & +\mathrm{DOX}\end{array}$ , $=-1-\quad \begin{aligned} & \text { Cleaved } \\ & \text { Caspase-3 }\end{aligned}$

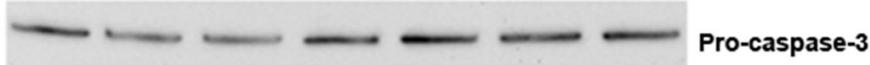

Cleaved caspase-3
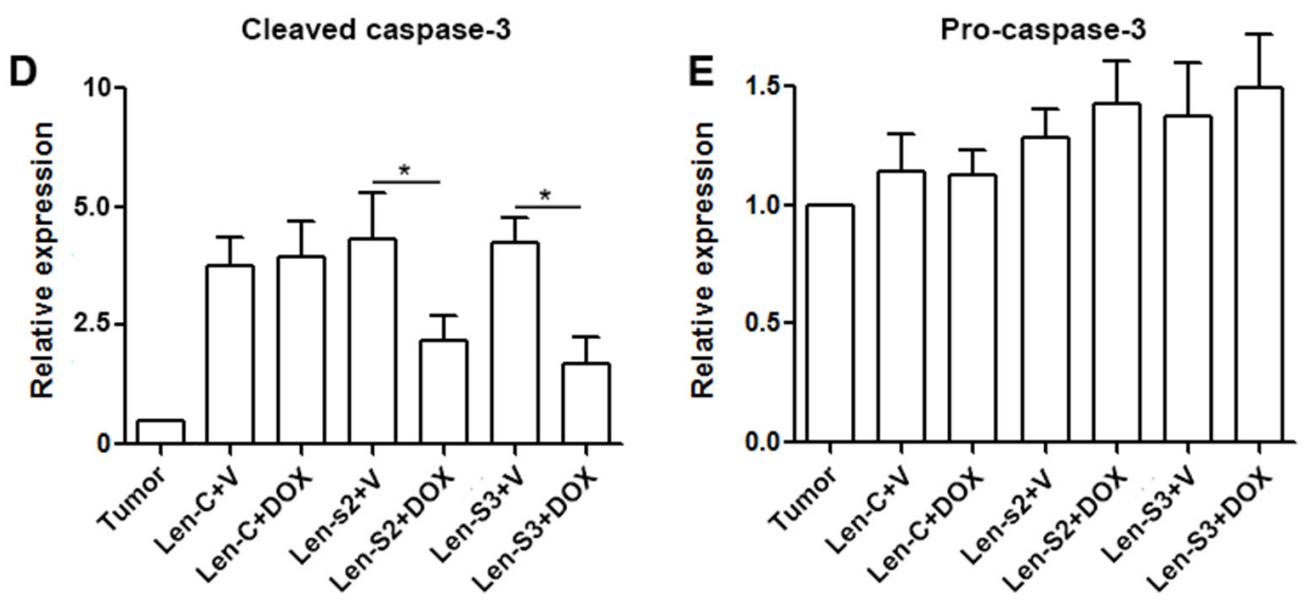

Figure 6: SESN2 and SESN3 suppresses NK-92 cell-mediated anti-ovarian cancer response in vivo. (A) Representative dot plots showing the proportions of EpCAM-expressing OVCAR-3 cells in peritoneal cells. No tumor: no tumor inoculation. Tumor: peritoneal tumor inoculation. Len-S2+V: transfer of Len-S2-transduced NK-92 cells with peritoneal PBS injections. Len-S2+DOX: transfer of Len-S2-transduced NK-92 cells with peritoneal doxycycline injections. Len-S3+V: transfer of Len-S3-transduced NK-92 cells with peritoneal PBS injections. Len-S3+DOX: transfer of Len-S3-transduced NK-92 cells with peritoneal doxycycline injections. (B) Absolute number of EpCAM-expressing OVCAR-3 cells harvested from mouse peritoneal cavity. N=7 per group. (C to E) Activation of caspase-3 in EpCAM-expressing OVCAR-3 cells isolated from mouse peritoneal cavity. Representative Western blot images were shown in (C). Statistics for cleaved caspase-3 was shown in (D). Statistics for pro-caspase-3 expression was shown in (E). N=3 per group. ", $p<0.05$; ${ }^{* * *}$, $\mathrm{p}<0.001$. 
NK-92 cells, the OVCAR-3 cell abundance was partially restored. Analysis of caspase-3, which is an apoptosis marker, showed less cleaved caspase- 3 in OVCAR-3 cells isolated from mice receiving doxycycline-treated NK-92 cells (Figure $6 \mathrm{C}$ to $6 \mathrm{E}$ ). Therefore, the tumoricidal activity of SESN2 or SESN3-overexpressing NK-92 cells was impaired in vivo.

\section{DISCUSSION}

SESNs are a family of highly conserved proteins that are induced upon various conditions of stress, including DNA damage and oxidative stress [12]. Physiologically, the activity of SESNs is modulated by nutrients including amino acids at both transcriptional and post-transcriptional levels. SESNs suppress oxidative damage through activation of Nrf2, autophagic removal of impaired mitochondria, and detoxification of reactive oxygen species via its peroxidase activity [12].

However, the significance of SESNs for immune cell development and functions have not been fully elucidated, probably due to their low expression levels in normal immune cells and organs. In this study we identified upregulation of SESN2 and SESN3 in intratumoral NK-92 cells, suggesting that the tumor microenvironment induced the expression of SESN2 and SESN3. However, the factors that induced SESN2 and SESN3 expression remain unclear. A previous research shows that $\mathrm{NO}$ and hypoxia up-regulate SESN2 in macrophages [28]. Indeed, hypoxic stress inside the tumor microenvironment impairs NK cell cytotoxicity [29], probably through inhibiting expression of activating receptors, granzyme B and perforin [30, 31]. Other factors such as mitochondria-dependent ROS production [32] and Toll-like receptor signaling [33] might also induce SESN expression. Interestingly, CD56, CD16 and CD158b are differentially expressed on NK cells in tumor-involved lymph nodes and uninvolved lymph nodes [34]. It will be necessary to check if their expression are intertwined with SESN expression.

The lentiviral system we used is a tetracycline inducible expression system in which SESN expression was induced by doxycycline, therefore avoiding immediate phenotypic and functional alterations of NK-92 cells after lentiviral transduction. We revealed that SESN2 and SESN3 suppressed tumoricidal activity of NK-92 cells. Notably, SESN2 or SESN3-induced phenotypic and functional changes of NK-92 cells were consistent with previously reported hypoxia-mediated changes of NK cells [30, 31]. Therefore, our data suggests the role of hypoxia in the up-regulation of SESNs. Interestingly, SESN3 seemed to be more potent in inhibiting NK-92 cell activity, suggesting that SESN3 might be more active than SESN2. In addition, whether SESN2 and SESN3 have mutual effects on one another needs to be studied in future. Moreover, it will be interesting to check whether normal NK cells up-regulate SESN expression in patients with ovarian cancer or other cancers.

The positive role of mTOR signaling, and the negative role of AMPK signaling in NK cell maturation and activation have been reported [35-37]. The effects of SESNs on mTOR and AMPK signaling have been disclosed in recent studies. SESNs inhibit mTORC1 kinase activation through direct interaction with GATOR2 [16], or through activation of AMPK and TSC [38]. Consistently, here we showed that SESNs-mediated AMPK signaling suppressed mTORC1 signaling in NK92 cells. Interestingly, the high level of activated AMPK $\beta 1$ in SESN-overexpressing NK-92 cells resulted from upregulation of AMPK $\beta 1$ protein, rather than up-regulation of phosphorylation. This data raises the possibility that SESNs might enhance AMPK $\beta 1$ expression. Indeed, it has been reported that SESN2 increases mRNA levels of AMPK $\alpha 1, \beta 1$ and $\gamma 1$ in breast cancer cells [38].

In conclusion, our data highlights the negative effects of SESN2 and SESN3 on NK-92 cell-mediated anti-ovarian cancer activity. Downregulating the expression of SESNs could be beneficial for NK-92 cellbased therapy against ovarian cancer.

\section{MATERIALS AND METHODS}

\section{Cells}

NK-92 cells and OVCAR-3 cells (ovarian cancer cell line) were purchased from Shanghai Baili Biotechnology Co., Ltd (Shanghai, China). NK-92 cells were cultured in $\alpha$-MEM (Gibco, Gaithersburg, MD, USA) with $10 \%$ fetal bovine serum (HyClone Laboratories, Logan, UT, USA), $100 \mathrm{U} / \mathrm{ml}$ penicillin/streptomycin (Gibco), and 100 U/ml recombinant hIL-2 (R\&D Systems, Minneapolis, MN, USA). OVCAR- 3 cells were cultured in RPMI-1640 Medium (Gibco) with 20\% fetal bovine serum and $100 \mathrm{U} /$ $\mathrm{ml}$ penicillin/streptomycin. Cells were subcultured every 2 days.

\section{Tumor xenograft models and NK-92 cell adoptive transfer}

Animal tests were approved by the Fujian Medical University Animal Care and Use Committee, and were conducted in accordance with institutional guidelines for animal use. Eight week old NOD/SCID/ $\gamma \mathrm{c}^{-/-}$mice (C57BL/6J background) were purchased from Beijing Vitalstar Biotechnology (Beijing, China). The subcutaneous tumor xenograft model was established by subcutaneous inoculation of $1 \times 10^{6}$ OVCAR- 3 cells on the left flank of each mouse. Thirty days later, NK-92 cells $\left(1 \times 10^{7}\right.$ cells in $100 \mu$ of PBS $)$ were injected into the peritoneum once a day for 2 days. Each mouse then received intraperitoneal injections of recombinant hIL2 (5 $\mu \mathrm{g}$ per mouse per day) for 6 days. At day 37 after 
inoculation, peritoneal cells were collected by rinsing the peritoneal cavity with $1 \mathrm{ml}$ of PBS. Spleens and tumor xenografts were mechanically dissociated on $70-\mu \mathrm{m}$ cell strainers to prepare single cell suspensions.

The intraperitoneal tumor xenograft model was conducted following previous reports with minor modifications [27, 39]. Briefly, mice were given $5 \times 10^{5}$ OVCAR-3 cells (in $50 \mu \mathrm{l}$ of PBS) via intraperitoneal injection 2 days prior to NK-92 cell injection. NK-92 cells $\left(5 \times 10^{6}\right.$ cells per mouse in $100 \mu$ of $\left.\mathrm{PBS}\right)$ were given to mice via intraperitoneal injection. Mice received hIL-2 as described above. Mice without NK-92 cell injection also received hIL-2. At day 6 after OVCAR-3 cell injection, cells in the peritoneal cavity were collected the same way as above.

\section{Flow cytometry and cells sorting}

The following antibodies were purchased from Biolegend (San Diego, California, USA): APC anti-CD45 (2D1), PE anti-CD56 (39D5), APC anti-EpCAM (9C4), Alexa Fluor ${ }^{\circledR} 647$ anti-granzyme B (GB11), PE/Cy7 antiPerforin (B-D48), Alexa Fluor ${ }^{\circledR} 647$ anti-NKp44 (P44-8), PE anti-NKG2D (1D11), PE anti-NKp46 (9E2), PE antiNKp30 (P30-15). Rabbit polyclonal anti-phospho-AMPK $\alpha$ (T183/T172) was purchased from Abcam (Cambridge, United Kingdom). PE/Cy7 anti-phospho-mTOR (S2448, clone\# MRRBY) was purchased from eBioscience (San Diego, California, USA). Alexa Fluor ${ }^{\circledR} 647$ polyclonal goat anti-rabbit IgG was purchased from Thermo Fisher (Shanghai, China). For cell surface marker staining, cells were incubated with $2 \mu \mathrm{g} / \mathrm{ml}$ antibody in PBS on ice for $15 \mathrm{~min}$. For cytokine detection, $10 \mu \mathrm{g} / \mathrm{mL}$ brefeldin A (Sigma-Aldrich, St. Louis, MO, USA) was added 3 hours before harvesting cells. Cells were then fixed with $4 \%$ paraformaldehyde for 15 minutes, permeabilized with $0.1 \%$ Triton $^{\mathrm{TM}} \mathrm{X}-100$ for 20 minutes, and incubated with $5 \mu \mathrm{g} / \mathrm{ml}$ cytokine antibodies for $1 \mathrm{~h}$ at room temperature. Cell apoptosis was analyzed using APC Annexin-V (eBioscience) following the vendor's manual. Apoptotic cells were identified as Annexin V-positive cells. Cells were analyzed on a BD LSRII flow cytometer. Cell sorting was performed on a BD Influx ${ }^{\mathrm{TM}}$ cell sorter.

\section{Lentiviral transduction}

Plasmids pRK5-FLAG-SESN2, pRK5-FLAGSESN3 and pCAG-GFP were purchased from Addgene (Cambridge, Massachusetts, USA). pLVX-TRE3G-IRES vector was purchased from Shanghai TranSheepBio Co., Ltd (Shanghai, China). The following primers were used to amplify human SESN2 or SESN3 coding sequences and EGFP sequence from these plasmids: SESN2 (5' -gg atccatgatcgtggcggactccgagtg-3' and 5'-gcggccgctcaggtc atgtagcgggtgatgg-3'); SESN3 (5' -ggatccatgaaccggggcgg 'ggcagc-3' and 5'-gcggccgctcaggtcaaatgccgagttatgg-3');
EGFP (5' -cccgggatggtgagcaagggcgagg-3' and 5' -gaattcct tgtacagctcgtccatgc-3'). Platinum ${ }^{\mathrm{TM}}$ Taq DNA Polymerase (Thermo Fisher) was used for PCR. SESN2 or SESN3 coding sequences were inserted into pLVX-TRE3G-IRES vector via digestion with restriction endonucleases BamHI and NotI, followed by ligation with T7 DNA Ligase. The new pLVX-TRE3G-SESN2-IRES and pLVX-TRE3GSESN3-IRES vectors were further ligated with EGFP sequence after digestion with SmaI and EcoRI. The resultant vectors were designated pLVX-TRE3G-SESN2IRES-EGFP and pLVX-TRE3G-SESN3-IRES-EGFP.

For packaging lentiviruses, $2.5 \times 10^{6}$ HEK293 cells were cultured with $25 \mu \mathrm{M}$ chloroquine diphosphate (Sigma-Aldrich). 0.72 pmol pRSV-Rev, 1.3 pmol pMDLg/ pRRE (both from Addgene), 1.64 pmol pLVX-TRE3GSESN2-IRES-EGFP or pLVX-TRE3G-SESN3-IRESEGFP were mixed with $83.4 \mu \mathrm{g}$ of PEI in $1 \mathrm{ml}$ of OptiMEM (Gibco). The mixture was then added to HEK293 cells for 18 hours before refreshing the medium. The viral supernatant was harvested 48 and 72 hours later. Lentivirus containing SESN2 or SESN3 sequence was designated Len-S2 and Len-S3, respectively. Lentivirus prepared using empty pLVX-TRE3G-IRES vector was designated Len-C. Packaging of lentivirus containing rtTA3 sequence (Termed Len-rtTA3) was conducted the same way as above, except that pLenti CMV rtTA3 Blast vector (Addgene) was used.

To transduce NK-92 cells, $1.0 \times 10^{5} / \mathrm{ml} \mathrm{NK}-92$ cells were stimulated with $100 \mathrm{U} / \mathrm{ml} \mathrm{hIL-2}$ and $100 \mathrm{ng} / \mathrm{ml}$ hIL-12 (Both from R\&D Systems) for 2 hours. Lentiviral particles were added to cells (Multiplicity of Infection was 30) with $8 \mu \mathrm{g} / \mathrm{ml}$ polybrene (Sigma-Aldrich) for 18 hours. Cells were then selected with $2 \mu \mathrm{g} / \mathrm{ml}$ puromycin (SigmaAldrich) for 6 days. Surviving cells were transduced with Len-rtTA3 in the same way, and were selected for additional 6 days with $2 \mu \mathrm{g} / \mathrm{ml}$ blasticidin (Sigma-Aldrich). Surviving cells were expanded for further experiments. To induce overexpression of exogenous genes in vitro, cells were treated with $200 \mathrm{ng} / \mathrm{ml}$ doxycycline (Sigma-Aldrich) for $48 \mathrm{~h}$. To induce expression in vivo, each mouse was intraperitoneally injected with $1 \mathrm{mg} / \mathrm{kg}$ doxycycline every two days.

\section{Cytotoxicity assay}

Lenitvirus-transduced NK-92 cells were pre-treated with or without $200 \mathrm{ng} / \mathrm{ml}$ doxycycline for $48 \mathrm{~h}$. These cells were then co-cultured with OVCAR-3 cells for $4 \mathrm{~h}$ at $37^{\circ} \mathrm{C}$ (effector : target $=10: 1$ ). Cell lysis was determined using LDH Cytotoxicity Assay Kit (Thermo Fisher), because LDH release is widely used in cytotoxicity assay [40]. OVCAR-3 apoptosis was distinguished by staining the whole cells with PE anti-CD56 antibody and APC Annexin-V, because OVCAR-3 cells do not express CD56. In some experiments, during the last 4 hours of 
doxycycline treatment, NK-92 cells were treated with10 $\mu \mathrm{M}$ compound $\mathrm{C}$ for 4 hours before co-culture.

\section{RNA isolation, reverse transcription and real- time quantitative PCR (q-PCR)}

RNA was isolated using the Eastep RNA Extraction Kit (Promega, Beijing, China). mRNA was transcribed into cDNA using VigoScript cDNA Synthesis Kit (Vigorous Biotechnology, Shanghai, China). q-PCR was performed using SYBR ${ }^{\circledR}$ Green Master Mix (Thermo Fisher) on a 7300 thermocycler (Invitrogen, Carlsbad, CA, USA). Primer sequences are as follows: TNF- $\alpha$ : 5'-ggagaagggtgaccgactca-3' and 5'-ctgcccagactcggcaa-3'. $\beta$-actin: 5'-tcacccacactgtgcccatctacg-3' and 5'-cagcggaacc gctcattgccaatg-3'.

\section{Western blot}

Proteins were extracted using RIPA buffer (Thermo Fisher). The following antibodies were used: anti- $\beta$-actinactin (sc-47778), anti-SESN2 (sc-101249), anti-phosphomTOR (S2448, sc-293133), anti-mTOR (sc-136269), anti-phospho-4EBP1 (S65, sc-293124), anti-4EBP1 (sc9977), anti-AMPK $\alpha 1 / 2$ (sc-74461), anti-AMPK $\beta 1$ (sc100357) and anti-caspase-3 (sc-7272) were purchased from Santa Cruz Biotechnology (Dallas, USA). AntiSESN1 (ab134091), anti-SESN3 (ab97792), anti-phosphoAMPK $\alpha 1 / 2$ (T183/T172, ab23875) and anti-phosphoAMPK $\beta 1$ (S181, ab55311) were purchased from Abcam. Cleaved caspase-3 (Asp175, MAB835) was purchased from R\&D Systems.

\section{Statistics}

Data were presented as mean \pm SD. All experiments were independently repeated for at least three times. Data was analyzed by one-way ANOVA followed by Fisher PLSD post hoc tests, or Student's t test. $P$ values $<0.05$ were considered significant.

\section{Author contributions}

XW, WL and JC performed most experiments and interpreted the data; DZ designed and performed the molecular cloning; SH performed several Western blot assay; WL conducted lentiviral packaging and transduction; XW designed the study and wrote the manuscript.

All authors have read and approved the final manuscript.

\section{CONFLICTS OF INTEREST}

The authors have no conflicts of interest to declare.

\section{FUNDING}

This study was supported by Fujian Provincial Natural Science Foundation (grant No. 2017J0105) and the Scientific Research Project of Department of Public Health of Fujian Province (grant No. 2012-2-52).

\section{REFERENCES}

1. Zhang L, Conejo-Garcia JR, Katsaros D, Gimotty PA, Massobrio M, Regnani G, Makrigiannakis A, Gray H, Schlienger K, Liebman MN, Rubin SC, Coukos G. Intratumoral $\mathrm{T}$ cells, recurrence, and survival in epithelial ovarian cancer. N Engl J Med. 2003; 348: 203-13.

2. Klingemann H, Boissel L, Toneguzzo F. Natural killer cells for immunotherapy - advantages of the NK-92 cell line over blood NK cells. Front Immunol. 2016; 7: 91.

3. Carlsten M, Childs RW. Genetic manipulation of NK cells for cancer immunotherapy: techniques and clinical implications. Front Immunol. 2015; 6: 266.

4. Tonn T, Becker S, Esser R, Schwabe D, Seifried E. Cellular immunotherapy of malignancies using the clonal natural killer cell line NK-92. J Hematother Stem Cell Res. 2001; 10: 535-44.

5. Klingemann HG. Natural killer cell-based immunotherapeutic strategies. Cytotherapy. 2005; 7: 16-22.

6. Malmberg KJ, Bryceson YT, Carlsten M, Andersson S, Bjorklund A, Bjorkstrom NK, Baumann BC, Fauriat C, Alici E, Dilber MS, Ljunggren HG. NK cell-mediated targeting of human cancer and possibilities for new means of immunotherapy. Cancer Immunol Immunother. 2008; 57 : 1541-52.

7. Lin A, Yan WH, Xu HH, Gan MF, Cai JF, Zhu M, Zhou MY. HLA-G expression in human ovarian carcinoma counteracts NK cell function. Ann Oncol. 2007; 18: 1804-9.

8. Xie J, Liu M, Li Y, Nie Y, Mi Q, Zhao S. Ovarian tumorassociated microRNA-20a decreases natural killer cell cytotoxicity by downregulating MICA/B expression. Cell Mol Immunol. 2014; 11: 495-502.

9. Uherek C, Tonn T, Uherek B, Becker S, Schnierle B, Klingemann HG, Wels W. Retargeting of natural killer-cell cytolytic activity to ErbB2-expressing cancer cells results in efficient and selective tumor cell destruction. Blood. 2002; 100: 1265-73.

10. Ruggeri L, Mancusi A, Capanni M, Martelli MF, Velardi A. Exploitation of alloreactive NK cells in adoptive immunotherapy of cancer. Curr Opin Immunol. 2005; 17: 211-7.

11. Belisle JA, Gubbels JA, Raphael CA, Migneault M, Rancourt C, Connor JP, Patankar MS. Peritoneal natural killer cells from epithelial ovarian cancer patients show an altered phenotype and bind to the tumour marker MUC16 (CA125). Immunology. 2007; 122: 418-29. 
12. Ho A, Cho CS, Namkoong S, Cho US, Lee JH. Biochemical basis of sestrin physiological activities. Trends Biochem Sci. 2016; 41: 621-32.

13. Budanov AV, Karin M. p53 target genes sestrin1 and sestrin 2 connect genotoxic stress and mTOR signaling. Cell. 2008; 134: 451-60.

14. Wolfson RL, Chantranupong L, Saxton RA, Shen K, Scaria SM, Cantor JR, Sabatini DM. Sestrin2 is a leucine sensor for the mTORC1 pathway. Science. 2016; 351: 43-8.

15. Tao R, Xiong X, Liangpunsakul S, Dong XC. Sestrin 3 protein enhances hepatic insulin sensitivity by direct activation of the mTORC2-Akt signaling. Diabetes. 2015; 64: 1211-23.

16. Parmigiani A, Nourbakhsh A, Ding B, Wang W, Kim YC, Akopiants K, Guan KL, Karin M, Budanov AV. Sestrins inhibit mTORC1 kinase activation through the GATOR complex. Cell Rep. 2014; 9: 1281-91.

17. Weichhart T, Hengstschlager M, Linke M. Regulation of innate immune cell function by mTOR. Nat Rev Immunol. 2015; 15: 599-614.

18. Keating R, McGargill MA. mTOR regulation of lymphoid cells in immunity to pathogens. Front Immunol. 2016; 7: 180.

19. Lanna A, Gomes DC, Muller-Durovic B, McDonnell T, Escors D, Gilroy DW, Lee JH, Karin M, Akbar AN. A sestrindependent Erk-Jnk-p38 MAPK activation complex inhibits immunity during aging. Nat Immunol. 2017; 18: 354-63.

20. Hu HJ, Shi ZY, Lin XL, Chen SM, Wang QY, Tang SY. Upregulation of Sestrin2 expression protects against macrophage apoptosis induced by oxidized low-density lipoprotein. DNA Cell Biol. 2015; 34: 296-302.

21. Kim MJ, Bae SH, Ryu JC, Kwon Y, Oh JH, Kwon J, Moon JS, Kim K, Miyawaki A, Lee MG, Shin J, Kim YS, Kim CH, et al. SESN2/sestrin2 suppresses sepsis by inducing mitophagy and inhibiting NLRP3 activation in macrophages. Autophagy. 2016; 12: 1272-91.

22. Bruning A, Rahmeh M, Friese K. Nelfinavir and bortezomib inhibit mTOR activity via ATF4-mediated sestrin-2 regulation. Mol Oncol. 2013; 7: 1012-8.

23. Shi X, Xu L, Doycheva DM, Tang J, Yan M, Zhang JH. Sestrin2, as a negative feedback regulator of $\mathrm{mTOR}$, provides neuroprotection by activation AMPK phosphorylation in neonatal hypoxic-ischemic encephalopathy in rat pups. J Cereb Blood Flow Metab. 2017; 37: 1447-60.

24. Xu J, Ji J, Yan XH. Cross-talk between AMPK and mTOR in regulating energy balance. Crit Rev Food Sci Nutr. 2012; 52: 373-81.

25. Li H, Liu S, Yuan H, Niu Y, Fu L. Sestrin 2 induces autophagy and attenuates insulin resistance by regulating AMPK signaling in C2C12 myotubes. Exp Cell Res. 2017; 354: 18-24.

26. Deng W, Cha J, Yuan J, Haraguchi H, Bartos A, Leishman E, Viollet B, Bradshaw HB, Hirota Y, Dey SK. p53 coordinates decidual sestrin 2/AMPK/mTORC1 signaling to govern parturition timing. J Clin Invest. 2016; 126: 2941-54.
27. Geller MA, Knorr DA, Hermanson DA, Pribyl L, Bendzick L, McCullar V, Miller JS, Kaufman DS. Intraperitoneal delivery of human natural killer cells for treatment of ovarian cancer in a mouse xenograft model. Cytotherapy. 2013; 15: 1297-306.

28. Essler S, Dehne N, Brune B. Role of sestrin2 in peroxide signaling in macrophages. FEBS Lett. 2009; 583: 3531-5.

29. Moretta L, Montaldo E, Vacca P, Del Zotto G, Moretta F, Merli P, Locatelli F, Mingari MC. Human natural killer cells: origin, receptors, function, and clinical applications. Int Arch Allergy Immunol. 2014; 164: 253-64.

30. Balsamo M, Manzini C, Pietra G, Raggi F, Blengio F, Mingari MC, Varesio L, Moretta L, Bosco MC, Vitale M. Hypoxia downregulates the expression of activating receptors involved in NK-cell-mediated target cell killing without affecting ADCC. Eur J Immunol. 2013; 43: 2756-64.

31. Sarkar S, Germeraad WT, Rouschop KM, Steeghs EM, van Gelder M, Bos GM, Wieten L. Hypoxia induced impairment of NK cell cytotoxicity against multiple myeloma can be overcome by IL-2 activation of the NK cells. PLoS One. 2013; 8: e64835.

32. Seo K, Seo S, Ki SH, Shin SM. Compound C increases sestrin2 expression via mitochondria-dependent ROS production. Biol Pharm Bull. 2016; 39: 799-806.

33. Kim MG, Yang JH, Kim KM, Jang CH, Jung JY, Cho IJ, Shin SM, Ki SH. Regulation of Toll-like receptor-mediated Sestrin2 induction by AP-1, Nrf2, and the ubiquitinproteasome system in macrophages. Toxicol Sci. 2015; 144: 425-35.

34. Vuletic A, Jurisic V, Jovanic I, Milovanovic Z, Nikolic S, Konjevic G. Distribution of several activating and inhibitory receptors on CD3(-)CD56(+) NK cells in regional lymph nodes of melanoma patients. J Surg Res. 2013; 183: 860-8.

35. Marcais A, Walzer T. mTOR: a gate to NK cell maturation and activation. Cell Cycle. 2014; 13: 3315-6.

36. Muller-Durovic B, Lanna A, Polaco Covre L, Mills RS, Henson SM, Akbar AN. Killer cell lectin-like receptor G1 inhibits NK cell function through activation of adenosine 5'-monophosphate-activated protein kinase. J Immunol. 2016; 197: 2891-9.

37. Kim KY, Kim JK, Han SH, Lim JS, Kim KI, Cho DH, Lee MS, Lee JH, Yoon DY, Yoon SR, Chung JW, Choi I, Kim $\mathrm{E}$, et al. Adiponectin is a negative regulator of NK cell cytotoxicity. J Immunol. 2006; 176: 5958-64.

38. Budanov AV, Lee JH, Karin M. Stressin' Sestrins take an aging fight. EMBO Mol Med. 2010; 2: 388-400.

39. Hermanson DL, Bendzick L, Pribyl L, McCullar V, Vogel RI, Miller JS, Geller MA, Kaufman DS. Induced pluripotent stem cell-derived natural killer cells for treatment of ovarian cancer. Stem Cells. 2016; 34: 93-101.

40. Jurisic V, Radenkovic S, Konjevic G. The actual role of LDH as tumor marker, biochemical and clinical aspects. Adv Exp Med Biol. 2015; 867: 115-24. 